\title{
Lyapunov Diagonal Semistability of Real H-Matrices
}

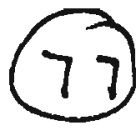

Daniel Hershkowitz and Hans Schneider*

Mathematics Department

University of Wisconsin, Madison

Madison, Wisconsin 53706

Dedicated in admiration to Helmut Wielandt on the occasion of his 75th birthday.

Submitted by Emeric Deutsch

\begin{abstract}
We characterize Lyapunov diagonally stable real $H$-matrices and those real $H$-matrices which are Lyapunov diagonally semistable but not Lyapunov diagonally stable (called Lyapunov diagonally near-stable). The latter characterization is given in terms of the principal submatrix rank property defined here. We apply our results to the numerical abscissas of real matrices. One of our main tools is a slight strengthening of classical results of Ostrowski which we derive from a fundamental theorem of Wielandt.
\end{abstract}

\section{INTRODUCTION}

A matrix $A \in \mathbb{R}^{n n}$ is called a $Z$-matrix if $A=s I-P, P \geqslant 0$ (entrywise), and an $M$-matrix if further $s \geqslant \rho(P)$, the spectral radius of $P$. A matrix $A \in \mathbb{C}^{n n}$ is called an H-matrix if the comparison matrix $M(A)$ is an $M$ matrix, where $B=M(A)$ is defined by $b_{i i}=\left|a_{i i}\right|, i=1, \ldots, n$, and $b_{i j}=$ $-\left|a_{i j}\right|, i \neq j, i, j=1, \ldots, n$. Because of their importance in applications there have been many papers on $H$-matrices, for example the recent one by Neumann and Plemmons [11].

A matrix $A \in \mathbb{R}^{n n}$ is said to be Lyapunov diagonally (semi)stable if there exists a positive diagonal matrix $D$, called a Lyapuno: scaling factor of $A$, such that $A D+D A^{T}$ is positive (semi)definite; see our previous paper [9]

*The research of this author was supported in part by NSF grant DMS-8320189. 
for some introductory remarks on such matrices. As in [9], it is also useful to call $A \in \mathbb{R}^{n n}$ Lyapunov diagonally near-stable if $A$ is Lyapunov diagonally semistable but not Lyapunov diagonally stable.

It is well known that Lyapunov diagonally stable [Lyapunov diagonally semistable] matrices are $P_{\text {-matrices }}\left[P_{0}\right.$-matrices], namely all their principal minors are positive [nonnegative]; see e.g. [7]. The problem of characterizing Lyapunov diagonally (semi)stable matrices is hard, and has been solved only in some special cases, for example for acyclic matrices by Berman and Hershkowitz [3]. By a well-known result (Araki and Kondo [1], Tartar [14; 4, p. 136]) a $Z$-matrix $A$ is a nonsingular $M$-matrix if and only if $A$ is Lyapunov diagonally stable. Berman, Varga, and Ward [5] extended the above result by characterizing Lyapunov diagonally semistable Z-matrices.

Theorem 0.1 [5, Theorem 1(i)]. Let $A \in \mathbb{R}^{n n}$ be an M-matrix. Then $A$ is Lyapunov diagonally semistable if and only if for each singular irreducible block $A_{i i}$ in the Frobenius normal form of $A$ we have $A_{i j}=0$ and $A_{j i}=0$, $i \neq j$.

(For definition of the Frobenius normal form see (4.1).)

In this paper we discuss Lyapunov diagonally stable and Lyapunov diagonally near stable (and therefore Lyapunov diagonally semistable) real $H$-matrices. Since a Lyapunov diagonally semistable matrix has nonnegative diagonal elements, it is natural to introduce the following definition:

Definition 0.2. Let $A \in \mathbb{R}^{n n}$. Then $A$ is an $H_{+}$-matrix if $A$ is an $H$-matrix and $a_{i i} \geqslant 0, i=1, \ldots, n$.

It is easy to prove that an $H_{+}$-matrix $A$ whose comparison matrix is nonsingular is Lyapunov diagonally stable (see also [2], [10, Chapter 10], where such matrices are called $H$-matrices). Here we first characterize Lyapunov diagonally stable $H_{+}$-matrices, and we then turn to the much harder characterization of Lyapunov diagonally near-stable $H_{+}$-matrices, which is one of our main goals.

We now describe our paper in detail. One of our major tools is Theorem 2.6, which is a slight strengthening of classical results due to Ostrowski [12] on determinants of $H$-matrices. We devote Section 2 to deriving this theorem from a fundamental result of Wielandt's [15] on conditions for the equality of $\rho(Q)$ and $\rho(P)$ where $P, Q \in \mathbb{C}^{n n}$ and $|Q| \leqslant P$, where $R=|Q|$ is defined by $r_{i j}=\left|q_{i j}\right|, i, j=1, \ldots, n$.

In Section 3 we show that an irreducible $H_{+}$-matrix $A$ is Lyapunov diagonally semistable (Proposition 3.1) and that $A$ is Lyapunov diagonally stable if and only if $A$ is nonsingular (Theorems 3.19 and 3.25). Thus an irreducible $H_{+}$-matrix $A$ is Lyapunov diagonally near-stable if and only if $A$ is 
singular. In Theorem 3.25 we give five other conditions that are equivalent. We also extend Theorem 2.6 in Theorem 3.13. We use a simple but basic lemma in [9] (Lemma 3.22 here) to show that the Lyapunov scaling factor of an irreducible $H_{+}$-matrix is unique up to positive scalar multiplication (Theorem 3.23).

In Section 4 we remove the condition of irreducibility. We show that an $H_{+}$-matrix $A$ is Lyapunov diagonally stable if and only if $A$ is nonsingular (Theorem 4.2). We introduce the main concepts of the paper, namely the principal submatrix rank property and the weak principal submatrix rank property (Definition 4.3). We show that an $H_{+}$-matrix $A$ is Lyapunov diagonally semistable if and only if $A$ has the principal submatrix rank property or, equivalently, the weak property (Theorem 4.14). Theorem 0.1 stated above now becomes a special case of Theorem 4.14. The rest of the section is devoted to a discussion of the implications in Theorem 4.14 in the case of general matrices, showing, in particular, that every Lyapunov diagonally semistable matrix has the weak principal submatrix rank property (Theorem 4.35).

In Section 5 we apply our results to the numerical abscissa $\sigma(A)$ of a real matrix $A$ (see Definition 5.1). We show that the infimum of $\sigma\left(\left(B+B^{T}\right) / 2\right)$ over real matrices $B$ which are diagonally similar to $A$ lies between $\sigma(A)$ and $\sigma(|A|)$ (Theorem 5.11). We discuss cases of equality in Theorem 5.11 in Theorems 5.15 and 5.16.

\section{NOTATION AND DEFINITIONS}

In this section we introduce some additional notation used in the sequel, as well as some definitions.

Notation 1.1.

$\langle n\rangle=$ the set $\{1,2, \ldots, n\}$, where $n$ is a positive integer.

$|\alpha|=$ the cardinality of the set $\alpha$.

$\operatorname{sign} a=\left\{\begin{array}{rr}1, & a>0 \\ -1, & a<0 \\ 0, & a=0\end{array}\right\}$, where $a$ is a real number.

Notation 1.2. Let $A \in \mathbb{R}^{n n}$ and let $\alpha, \beta \subseteq\langle n\rangle, \alpha, \beta \neq \varnothing$. We denote:

$N(A)=$ the null space of $A$.

span $(A)=$ the subspace of $\mathbb{R}^{n}$ which is spanned by the columns of $A$. 
$A[\alpha \mid \beta]=$ the submatrix of $A$ whose rows are indexed by $\alpha$ and whose columns are indexed by $\beta$ in their natural orders. $A[\alpha]=A[\alpha \mid \alpha]$. $A(\alpha)=A[\langle n\rangle \backslash \alpha]$, where $\alpha \neq\langle n\rangle$.

REMARK. Similar notation will be used also for complex matrices.

\section{DeFinition 1.3.}

(i) A real $n \times n$ matrix $A$ is said to be symmetric in sign if $a_{i j} a_{j i} \geqslant 0$, $1 \leqslant i, j \leqslant n$.

(ii) Let $A, B \in \mathbb{R}^{n n}$. The matrices $A$ and $B$ are said to be sign similar if there exists a real diagonal $n \times n$ matrix $E$ such that $|E|=I$ satisfying $A=E B E$.

(iii) A real $n \times n$ matrix $A$ is said to be row diagonally dominant if

$$
a_{i i} \geqslant \sum_{\substack{j=1 \\ j \neq i}}^{\prime \prime}\left|a_{i j}\right|, \quad 1 \leqslant i \leqslant n .
$$

The matrix $A$ is said to be column diagonally dominant if $A^{T}$ is row diagonally dominant.

(iv) A row diagonally dominant matrix $A$ is said to be strictly row diagonally dominant if strict inequalities hold in (1.4), and similarly for strict column diagonal dominance.

(v) Let $A=s I-P, P \geqslant 0, s$ real, be a $Z$-matrix. The minimal eigenvalue of $A$ is defined to be $s-\rho(P)$.

REMARK 1.5. Whenever we say "positive [semi]definite matrix" in this paper we mean "positive [semi]definite hermitian matrix".

\section{ON RESULTS OF OSTROWSKI'S AND WIELANDT'S}

Our basic tools are results on determinants essentially due to Ostrowski [12]; see Satz I, Zusatz zu Satz I, and Satz V. Since in the sequel these results are applied in a form slightly different from that found in [12], we shall derive them from a version of the Perron-Frobenius theorem which is due to Wielandt [15]. We begin by stating Wielandt's result, omitting an assumption of irreducibility where it is superfluous. 
TheOREM $2.1[15, \mathrm{III}]$. Let $P$ be a nonnegative matrix in $\mathbb{R}^{n n}$ and let $Q \in \mathbb{C}^{n n}$. Let $|Q| \leqslant P$. Then $\rho(Q) \leqslant \rho(P)$.

Further, if $P$ is irreducible then the following are equivalent:

(i) $\rho(Q)=\rho(P)$.

(ii) $Q=e^{i \phi} D^{-1} P D$ where $\varphi$ is real and $|D|=I$.

Corollary 2.2. Let $A$ be an $H_{+}$-matrix in $\mathbb{R}^{n n}$, and let $B$ be an $M$-matrix in $\mathbb{R}^{n n}$ such that $M(A) \geqslant B$. Let $\beta$ be the minimal eigenvalue of $B$. Then

$$
\operatorname{Re} \lambda \geqslant \beta \quad \text { for every } \lambda \in \operatorname{spec}(A) .
$$

Further, if $B$ is irreducible then the following are equivalent:

(i) $\operatorname{Re} \lambda=\beta$ for some $\lambda \in \operatorname{spec}(A)$,

(ii) $\lambda=\beta$ for some $\lambda \in \operatorname{spec}(A)$,

(iii) $A$ is sign similar to $B$.

Proof. Let $s>a_{i i}, i=1, \ldots, n$, and write

$$
B=s I-P
$$

and

$$
A=s I-Q .
$$

Observe that $P \geqslant 0$ and that $M(A)=s I--|Q|$, since $A$ is an $H_{+}$-matrix. Obviously, $M(A) \geqslant B$ implies that $P \geqslant|Q|$. "The first part of the theorem now follows from Theorem 2.1. For the second part observe that (iii) $\Rightarrow$ (ii) and (ii) $\Rightarrow$ (i) are in mecliate.

We shall prove (i) $\Rightarrow$ (iii). If (i) holds then $\rho(Q) \geqslant \rho(P)$. Hence, by Theorem 2.1, $o(Q)=\rho(P)$ and there exist $\varphi$ and a diagonal matrix $D$ such that $|D|=I$ and $Q=e^{i \Phi} D^{-1} P D$. Since the diagonal entries of $P$ and $Q$ are positive, it follows that $e^{i \varphi}=1$. Let $D=\operatorname{diag}\left\{d_{1}, \ldots, d_{n}\right\}$. Without loss of generality we may assume that

$$
d_{1}=1
$$

Since $B$ is irreducible, for each $i, 2 \leqslant i \leqslant n$, there exists a sequence $t_{1}, \ldots, t_{k}$ such that $t_{1}=1, t_{k}=i$, and $p_{t_{l} t_{1+1}} \neq 0, l=1, \ldots, k-1$. Note that

$$
\prod_{l=1}^{k-1} q_{l, l_{i+1}}=\frac{d_{i}}{d_{1}} \prod_{l=1}^{k-1} p_{l, l_{t+1}}
$$


Since $P$ and $Q$ are real, it follows from (2.4), (2.5), and $|D|=I$ that $d_{i}= \pm 1$. Thus $P$ and $Q$ are sign similar, and the sign similarity of $A$ and $B$ follows.

Theorem 2.6. Let $A$ be an $H_{+}$-matrix in $\mathbb{R}^{n n}$ and let $B$ be an M-matrix in $\mathbb{R}^{\prime \prime \prime}$. Suppose $M(A) \geqslant B$. Then

$$
\forall \alpha \subseteq\langle n\rangle, \quad \operatorname{det} A[\alpha] \geqslant \operatorname{det} B[\alpha] \geqslant 0 .
$$

Further, if $B$ is irreducible then the following are equivalent:

(i) $A$ is sign similar to $M(A)$, and $M(A)=B$.

(ii) For all $\alpha \subseteq\langle n\rangle$, $\operatorname{det} A[\alpha]=\operatorname{det} B[\alpha]$.

(iii) $\operatorname{det} B=\operatorname{det} A$.

Proof. Since $A[\alpha], B[\alpha]$ satisfy the hypotheses of this theorem for every $\alpha \subseteq\langle n\rangle$, it is enough to prove (2.7) for the case $\alpha=\langle n\rangle$.

We first consider the case when $B$ is singular. By (2.3), $\operatorname{Re} \lambda \geqslant 0$ for each $\lambda \in \operatorname{spec}(A)$. Since $A$ is real, it follows that $\operatorname{det} A \geqslant 0$ and (2.7) is proved.

Now suppose that $B$ is irreducible. Clearly (i) $\Rightarrow$ (ii) and (ii) $\Rightarrow$ (iii). Suppose that (iii) holds. Then (ii) of Corollary 2.2 is satisfied, since $\beta=0$. Condition (i) now follows by Corollary 2.2 .

We prove the general case by induction. If $n=1$, the result is trivial. Inductively, we assume that (2.7) holds provided that $n<k$. Now let $n=k$. Let $t \geqslant 0$ be such that $B^{\prime}=B-t I$ is a singular $M$-matrix and let $A^{\prime}=A-t I$. Then $A^{\prime}$ is an $H_{+}$-matrix and $M\left(A^{\prime}\right) \geqslant B^{\prime}$. We have

$$
\begin{aligned}
& \operatorname{det} A=\sum_{\varnothing \subseteq \alpha \subseteq\langle n\rangle} t^{|\alpha|} \operatorname{det} A^{\prime}(\alpha), \\
& \operatorname{det} B=\sum_{\varnothing \subseteq \alpha \subseteq\langle n\rangle} t^{|\alpha|} \operatorname{det} B^{\prime}(\alpha),
\end{aligned}
$$

where $\operatorname{det} A(\langle n\rangle)=1$. By our inductive assumption, $\operatorname{det} A^{\prime}(\alpha) \geqslant \operatorname{det} B^{\prime}(\alpha) \geqslant 0$ : and $\operatorname{det} A^{\prime} \geqslant 0$, as shown above. Hence (2.7) follows.

To prove the equivalence of (i), (ii), and (iii), again observe that (i) $\Rightarrow$ (ii) and (ii) $\Rightarrow$ (iii) are immediate. For the proof of (iii) $\Rightarrow$ (i), note that by (2.8) and (2.9) $\operatorname{det} B=\operatorname{det} A$ implies $\operatorname{det} B^{\prime}=\operatorname{det} A^{\prime}$. Hence (i) follows by the singular case. 


\section{IRREDUCIBLE $H_{+}$-MATRICES}

We begin with a known proposition which generalizes results of Tartar [14] and Araki and Kondo [1]. Our proof is due to C. R. Johnson (private communication).

Propositron 3.1. If $A \in \mathbb{R}^{n 11}$ is an irreducible $H_{+}$matrix, then $A$ is Lyapunov diagonally semistable. If, further, $M(A)$ is nonsingular, then $A$ is Lyapunov diagonally stable.

Proof. As is well known, there exist positive diagonal matrices $E$ and $D$ such that $E M(A) D$ is both row and column diagonally dominant (e.g. [4, p. 156]). Clearly, the same holds for $E A D$, and hence the matrix $C=E A D+$ $D A^{T} E$ is positive semidefinite, since it is diagonally dominant. Thus, the matrix $E^{-1} C E^{-1}=A D E^{-1}+E^{-1} D A$ is positive semidefinite, and so $A$ is Lyapunov diagonally semistable.

In the case that $M(A)$ is nonsingular then $E M(A) D$ may be chosen to be strictly row and column diagonally dominant (e.g. [4, p. 136]), and the rest of the proof follows as above.

We shall strengthen the second part of Proposition 3.1 in Theorem 4.2 by replacing the hypothesis that $M(A)$ is nonsingular with the hypothesis that $A$ is nonsingular. This is a weaker condition by Theorem 2.6 .

The converse of Proposition 3.1 is false; namely, a Lyapunov diagonally semistable matrix is not necessarily an $H_{+}$-matrix, as demonstrated by the following example. Let

$$
A=\left[\begin{array}{ccc}
1 & a & a \\
-a & 1 & a \\
-a & -a & 1
\end{array}\right]
$$

where $a$ is any real number. Here $A$ is even Lyapunov diagonally stable, since $A+A^{T}=2 I$. But $M(A)$ is not an $M$-matrix for $|a|>\frac{1}{2}$.

In the sequel we determine which $H_{+}$-matrices are Lyapunov diagonally stable and which are not, but are Lyapunov diagonally semistable.

We start with two lemmas.

Lemma 3.2. Let $A, B \in \mathbb{B}^{n n}$ such that $B$ is a Z-matrix and $M(A) \geqslant B$. Then

$$
M\left(A+A^{T}\right) \geqslant B+B^{T} .
$$


Equality in (3.3) holds if and only if $M(A)=B$ and $A$ is symmetric in sign.

Proof. Since $M(A) \geqslant B$, we have

$$
M\left(A+A^{T}\right)_{i i}=2\left|a_{i i}\right| \geqslant 2 b_{i i}=\left(B+B^{T}\right)_{i i}, \quad i=1, \ldots, n,
$$

and for $i \neq j, i, j=1, \ldots, n$,

$$
\begin{aligned}
M\left(A+A^{T}\right)_{i j} & =-\left|a_{i j}+a_{j i}\right| \\
& \geqslant-\left|a_{i j}\right|-\left|a_{j i}\right|=M(A)_{i j}+M(A)_{j i} \\
& \geqslant b_{i j}+b_{j i}=\left(B+B^{T}\right)_{i j} .
\end{aligned}
$$

Observe that equalities in (3.4) and (3.5) hold if and only if $M(A)=B$ and $A$ is symmetric in sign.

The conditions $M(A) \geqslant B$ and the sign similarity of $A$ and $B$ will appear frequently in the sequel. We shall show the sign similarity of $A$ and $B$ is equivalent to the diagonal similarity of $A$ and $B$ under certain conditions. The next two lemmas are not used in subsequent proofs.

Lemma 3.6. Let $A, B \in \mathbb{R}^{n n}$ be such that $\left|a_{i j}\right| \leqslant\left|b_{i j}\right|, i \neq j, i, j=$ $1, \ldots, n$. If $B$ is irreducible and $D$ is a nonsingular diagonal matrix in $\mathbb{R}^{n n}$ such that $D^{-1} B D=A$, then $|D|=c I$.

Proof. If $|D| \neq c I$, then without loss of generality we may assume that $\left|d_{1}\right|=\left|d_{2}\right|=\cdots=\left|d_{k}\right|>\left|d_{k+1}\right| \geqslant\left|d_{k+2}\right| \geqslant \cdots \geqslant\left|d_{n}\right|$. Since $B$ is irreducible, there exist $i, j, k<i \leqslant n, 1 \leqslant j \leqslant k$, such that $b_{i j} \neq 0$. Then

$$
\left|a_{i j}\right|=\left|d_{i}^{-1} b_{i j} d_{j}\right|>\left|b_{i j}\right|
$$

contrary to assumption.

A lemma of the same flavor is the following.

Lemma 3.7. Let $A, B \in \mathbb{R}^{n n}$ be matrices such that $|A|,|B|$ are symmetric. If $B$ is irreducible and $D$ is a nonsingular diagonal matrix in $\mathbb{R}^{n n}$ such that $A=D^{-1} B D$, then $|D|=c I$. 
Proof. If $|D| \neq c I$, then, as in the proof of the previous lemma, we may assume that $\left|d_{1}\right|=\cdots=\left|d_{k}\right|>\left|d_{k+1}\right| \geqslant \cdots \geqslant\left|d_{n}\right|$. There exist $i, j, k<i \leqslant$ $n, 1 \leqslant j \leqslant k$ such that $\left|b_{i j}\right|=\left|b_{j i}\right| \neq 0$. Then

$$
\begin{aligned}
& \left|a_{i j}\right|=\left|d_{i} b_{i j} d_{j}^{-1}\right|<\left|b_{i j}\right|, \\
& \left|a_{j i}\right|=\left|d_{j} b_{j i} d_{i}^{-1}\right|>\left|b_{j i}\right|,
\end{aligned}
$$

which is a contradiction.

Similar results hold for complex matrices.

Lemma 3.8. Let $A, B \in \mathbb{R}^{n n}$ such that $B$ is a Z-matrix and $M(A) \geqslant B$. Then $A$ is sign similar to $B$ if and only if $A+A^{T}$ is sign similar to $B+B^{T}$.

Proof. If $A$ is sign similar to $B$, then $A=E B E$, where $E$ is a real diagonal matrix with $|E|=I$. Hence $A^{T}=E B^{T} E$, and so $A+A^{T}$ and $B+B^{T}$ are sign similar.

Conversely, suppose

$$
A+A^{T}=E\left(B+B^{T}\right) E .
$$

Clearly,

$$
M\left(A+A^{T}\right)=B+B^{T} .
$$

By Lemma 3.2, since $M(A) \geqslant B,(3.10)$ implies that $M(A)=B$ and that $A$ is symmetric in sign. Let $i \neq j, 1 \leqslant i, j \leqslant n$. Then either $a_{i j}=e_{i} b_{i j} e_{j}$ or $a_{i j}$ $=-e_{i} b_{i j} e_{j}$. Assume that for some $i, j, i \neq j$, we have $a_{i j} \neq e_{i} b_{i j} e_{j}$. Then necessarily

$$
a_{i j}=-e_{i} b_{i j} e_{j} \neq 0 .
$$

Since $B$ is an $M$-matrix, and $A$ is symmetric in sign, we have

$$
a_{j i}=-e_{j} b_{i j} e_{i} .
$$

Hence

$$
\left(A+A^{T}\right)_{i j}=-\left[E\left(B+B^{T}\right) E\right]_{i j},
$$

and by (3.9) this implies that

$$
a_{i j}=a_{j i}=b_{i j}=b_{j i}=0,
$$


which is a contradiction to (3.11). Hence $A=E B E$.

TheOREM 3.13. Let $B \in \mathbb{R}^{n n}$ be an M-matrix such that $B+B^{T}$ is positive semidefinite, and let $A \in \mathbb{R}^{n n}$ be an $H_{+}$-matrix satisfying $M(A) \geqslant B$. Then

$$
\forall \alpha \subseteq\langle n\rangle, \quad \operatorname{det}\left(A+A^{T}\right)[\alpha] \geqslant \operatorname{det}\left(B+B^{T}\right)[\alpha] .
$$

Furthermore, if $B$ is irreducible, then the following are equivalent:

(i) Equality holds in (3.14),

(ii) $\forall \alpha \subseteq\langle n\rangle, \operatorname{det} A[\alpha]=\operatorname{det} B[\alpha]$,

(iii) $\operatorname{det}\left(A+A^{T}\right)=\operatorname{det}\left(B+B^{T}\right)$,

(iv) $\operatorname{det} A=\operatorname{det} B$,

(v) $A+A^{T}$ is sign similar to $B+B^{T}$,

(vi) $A$ is sign similar to $B$.

Proof. By Lemma 3.2, we have

$$
M\left(A+A^{T}\right) \geqslant B+B^{T} .
$$

Sine $B+B^{T}$ is a positive semidefinite Z-matrix, it is an $M$-matrix. Hence by Theorem 2.6 we obtain (3.14).

If $B$ is irreducible then so is $B+B^{T}$, since $B$ is a $Z$-matrix. By Theorem 2.6, concitions (i), (iii), anci (v) are equivalent, as well as conditions (ii), (iv), and (vi). Conditions (v) and (vi) are equivalent by Lemma 3.8.

As a consequence of Theorem 3.13 we get

Proposition 3.16. Let $A$ be an $H_{+}$-matrix, and let $B$ be an irreducible $M$-matrix such that $M(A) \geqslant B$. Then $A$ is Lyapunov diagonally semistable.

Furthermore, the following are equivalent:

(i) A is Lyapunov diagonally near-stable.

(ii) $A$ is sign similar to $B$, and $B$ is singular.

(iii) $A$ is singular.

Proof. The first part of the proposition was already proved in Proposi- . tion 3.1. We give a simple second proof which uses the previous results. By [5] there exists a positive diagonal matrix $D$ such that $B D+D B^{T}$ is positive semidefinite and thus an $M$-matrix. We now apply Theorem 3.13 to $B D$ and 
$A D$, and we obtain

$$
\forall \alpha \subseteq\langle n\rangle, \quad \operatorname{det}\left(A D+D A^{T}\right)[\alpha] \geqslant \operatorname{det}\left(B D+D B^{T}\right)[\alpha] \geqslant 0
$$

Hence, $A$ is Lyapunov diagonally semistable.

We now prove the equivalence of (i), (ii), and (iii).

(i) $\Rightarrow$ (ii): Since $A$ is Lyapunov diagonally near-stable, it follows from (3.17) that

$$
\operatorname{det}\left(A D+D A^{T}\right)=\operatorname{det}\left(B D+D B^{r}\right)=0
$$

Since $B$ is irreducible, it follows from Theorem 3.13 that $A D$ and $B D$ are sign similar. Hence $A$ and $B$ are sign similar. Assume now that $B$ is nonsingular. By [I], the matrix $D$ at the beginning of the proof may be chosen such that $B D+D B^{r}$ is positive definite, which is a contradiction to (3.18). Hence, $B$ is singular.

(ii) $\Rightarrow$ (iii): Obvious.

(iii) $\Rightarrow$ (i): Since Lyapunov diagonally stable matrices have positive principal minors (e.g. [7]), and since, as proved above, $A$ is Lyapunov diagonally semistable, it follows from (iii) that $A$ is necessarily Lyapunov diagonally near-stable.

By applying Proposition 3.16 to $A$ and $B=M(A)$ we obtain the following theorem, which completes Proposition 3.1 by characterizing those irreducible $H_{+}$-matrices which are Lyapunov diagonally near-stable.

Theorem 3.19. Let $A$ be an irreducible $H_{+}$matrix. Then $A$ is Lyapunov diagonally semistable.

Furthermore, the following are equivalent:

(i) $A$ is Lyapunov diagonally near-stable.

(ii) $A$ is sign similar to $M(A)$, and $M(A)$ is singular.

(iii) A is singular.

Theorem 3.19 does not hold for reducible $H_{+}$-matrices, as demonstrated by the singular matrix

$$
A=\left[\begin{array}{rrr}
1 & -1 & 1 \\
-1 & 1 & -2 \\
0 & 0 & 1
\end{array}\right]
$$

The matrix $A$ is not Lyapunov diagonally semistable, since it does not satisfy the conditions of Theorem 4.12 of the next section. It is also easy to 
verify that $A$ and $M(A)$ are not sign similar. Those reducible $H_{+}$-matrices which are Lyapunov diagonally semistable will be characterized in the next section.

As observed in [9], the Lyapunov scaling factor of a Lyapunov diagonally stable matrix is not unique if the order is greater than 1 . We now show that the Lyapunov scaling factor of a singular irreducible $H_{+}$-matrix is unique (up to a positive scalar multiplication).

Lemma 3.20. Let $A \in \mathbb{R}^{n n}$ be a singular irreducible $H_{+}$-matrix. Then all the principal minors of $A$ of order less than $n$ are positive.

Proof. Observe that $M(A)$ is an irreducible $M$-matrix. As such, it is well known that all the principal minors of $M(A)$ of order less than $n$ are positive. Our claim now follows from Theorem 2.6.

Recall that the null space of an $n \times n$ matrix $A$, denoted by $N(A)$, is defined to be the set of all vectors $x \in \mathbb{C}^{n}$ such that $A x=0$.

Lemma 3.21. Let $A \in \mathbb{R}^{n n}$ be a singular irreducible $H_{+}-$matrix. Then there exists a unique diagonal $n \times n$ matrix $D$ such that $N(A D)=N\left(A^{T}\right)$. [9].

Proof. The lemma follows from Lemma 3.20 and from Corollary 5.5 of

The following Lemma is Lemma 6.6 of [9]. This lemma is going to be used several times in the sequel, and thus we quote it here.'

Lemma 3.22. Let $A$ be a singular Lyapunov diagonally semistable matrix, and let $D$ be a Lyapunov scaling factor of $A$. Then

$$
N(A D)=N\left(A^{T}\right) \subseteq N\left(A D+D A^{T}\right)
$$

Theorem 3.23. Let $A \in \mathbb{R}^{n n}$ be a singular irreducible $H_{+}-$matrix. Then $A$ has a unique Lyapunov scaling factor.

Proof. Let $D$ be a Lyapunov scaling factor of $A$. By Lemma 3.22, $N(A D)=N\left(A^{T}\right)$. The uniqueness of $D$ now follows from Lemma 3.21.

REMark 3.24. Theorem 3.23 does not hold for reducible $H_{+}$-matrices. By Theorem 6.20 of [9] the Lyapunov scaling factor of any reducible Lyapunov diagonally semistable matrix is not unique. 
We conclude the section with a theorem which summarizes the results of this section.

Theorem 3.25. Let $A$ be an irreducible $H_{+}$-matrix of order greater than 1. Then $A$ is Lyapunov diagonally semistable.

Furthermore, the following are equivalent:

(i) A is Lyapunov diagonally near stable.

(ii) $A$ is sign similar to $M(A)$, and $M(A)$ is singular.

(iii) $A$ is singular.

(iv) A has a unique Lyapunov scaling factor.

(v) There exists a unique diagonal matrix $D$ such that $N(A D)=N\left(A^{T}\right)$.

(vi) There exists a unique positive diagonal matrix $D$ such that $N(A D)=$ $N\left(A^{T}\right)$.

(vii) There exists a positive diagonal matrix $D$ such that $N(A D)=N\left(A^{T}\right)$ $\neq\{0\}$, and each such matrix $D$ is a Lyapunov scaling factor of $A$.

Proof. In view of Theorem 3.19, we just have to prove the equivalence of (iv), (v), (vi), and (vii) to any of (i), (ii), and (iii).

(iii) $\Rightarrow$ (iv) by Theorem 3.23 .

(iv) $\Rightarrow$ (i): As observed above, since $A$ has a unique Lyapunov scaling factor, and since the order of $A$ is greater than $1, A$ is necessarily Lyapunov diagonally near-stable.

(iii) $\Rightarrow$ (v) by Lemma 3.21 .

(v) $\Rightarrow$ (iii): Since there exists a unique diagonal matrix $D$ such that $N(A D)=N\left(A^{T}\right)$, and since the order of $A$ is greater than 1 , it follows that $N\left(A^{T^{*}}\right) \neq\{0\}$.

(iv) $\Rightarrow$ (vi): By Lemma 3.22, the Lyapunov scaling factor $D$ of $A$ is a positive diagonal matrix satisfying $N(A D)=N\left(A^{T}\right)$. The uniqueness follows from (v), which has been proved to be equivalent to (iv).

(vi) $\Rightarrow$ (vii): $N\left(A^{T}\right) \neq\{0\}$, since there is a unique positive diagonal matrix $D$ such that $N(A D)=N\left(A^{T}\right)$. This matrix is necessarily the Lyapunov scaling factor of $A$ by Lemma 3.22.

(vii) $\Rightarrow$ (iii): The matrix $A$ is singular, since $N\left(A^{T}\right) \neq\{0\}$.

\section{REDUCIBLE $H_{+}$-MATRICES}

Let $A$ be an $n \times n H_{+}$-matrix. Since Lyapunov diagonal stability and the property of being an $H_{-1}$-matrix are not affected by identical permutation of 
rows and columns, we may assume that $A$ is in Frobenius normal form

$$
A=\left[\begin{array}{cccc}
A_{11} & A_{12} & \cdots & A_{1 k} \\
0 & A_{22} & \cdots & A_{2 k} \\
\vdots & & \ddots & \cdots \\
0 & \cdots & 0 & A_{k k}
\end{array}\right]
$$

where $A_{i i}, i=1, \ldots, k$, is a square irreducible matrix.

As mentioned in the previous section, a reducible $H_{+}$-matrix is not necessarily Lyapunov diagonally semistable. In this section we determine which $H_{+}$-matrices are Lyapunov diagonally semistable. We begin with a theorem which characterizes the Lyapunov diagonally stable matrices.

Theorem 4.2. Let $A$ be an $H_{+}$-matrix. Then $A$ is Lyapunov diagonally stable if and only if $A$ is nonsingular.

Proof. Let the matrix $A$ be given in the form (4.1). As shown in [3], $A$ is Lyapunov diagonally stable if and only if each $A_{i i}$ is Lyapunov diagonally stable. The assertion now follows from Theorem 3.19.

The characterization of Lyapunov diagonally semistable $M$-matrices contained in Theorem 0.1 does not hold for $H_{+}$-matrices. For example consider the $H_{+}$-matrix

$$
A=\left[\begin{array}{lll}
1 & 1 & 2 \\
1 & 1 & 2 \\
0 & 0 & 1
\end{array}\right]
$$

which is Lyapunov diagonally semistable, since $A+A^{T}$ is positive semidefinite. We shall, however, generalize the result of [2] to $H_{+}$-matrices.

Definition 4.3. An $n \times n$ matrix $A$ is said to have the principal submatrix rank property if it satisfies

$$
\operatorname{rank} A[\alpha \mid\langle n\rangle]=\operatorname{rank} A[\langle n\rangle \mid \alpha]=\operatorname{rank} A[\alpha]
$$

for all nonempty sets $\alpha \subseteq\langle n\rangle$. 
The matrix $A$ is said to have the weak principal submatrix rank property if it satisfies

$$
\left\{\begin{array}{ccc}
A[j \mid \alpha]=0 & \Rightarrow & A[\alpha \mid j] \in \operatorname{span}(A[\alpha]), \\
A[\alpha \mid j]=0 & \Rightarrow & A[j \mid \alpha]^{T} \in \operatorname{span}\left(A[\alpha]^{T}\right)
\end{array}\right\} \text { for all } j \in\langle n\rangle \backslash \alpha,
$$

for all nonempty sets $\alpha \subseteq\langle n\rangle$.

Proposition 4.6. Let $A$ be an $n \times n$ matrix. Then $A$ has the principal submatrix rank property if and only if (4.4) holds for all sets $\alpha$ such that $A[\alpha]$ is irreducible and singular.

Proof. The "only if" part is obvious. Conversely, it is clear that (4.4) holds for nonsingular irreducible principal submatrices, so we may now assume it holds for all irreducible principal submatrices. Let $B=A[\alpha]$ be any principal submatrix of $A$. Without loss of generality, we may assume that $B$ has the form

$$
B=\left[\begin{array}{cccc}
B_{11} & B_{12} & \ldots & B_{1 r} \\
0 & B_{22} & \ldots & B_{2 r} \\
\vdots & \ddots & \ddots & \vdots \\
0 & \cdots & 0 & B_{r r}
\end{array}\right]
$$

where $B_{i i}, i=1, \ldots, r$, is a square irreducible matrix. We prove our claim by induction on $r$. For $r=1$ there is nothing to prove, since $B$ is irreducible. Assume that our claim holds for $r<m$, and let $r=m$. Let $\beta \subseteq\langle n\rangle$ be the set such that

$$
B_{11}=A[\beta]
$$

By the inductive assumption

$$
\operatorname{rank} A[\alpha \backslash \beta \mid\langle n\rangle]=\operatorname{rank} A[\langle n\rangle \mid \alpha \backslash \beta]=\operatorname{rank} A[\alpha \backslash \beta]
$$

and

$$
\operatorname{rank} A[\beta \mid\langle n\rangle]=\operatorname{rank} A[\langle n\rangle \mid \beta]=\operatorname{rank} A[\beta]
$$


Hence,

$$
\begin{aligned}
\operatorname{rank} A[\alpha \mid\langle n\rangle] & \leqslant \operatorname{rank} A[\alpha \backslash \beta \mid\langle n\rangle]+\operatorname{rank} A[\beta \mid\langle n\rangle] \\
& =\operatorname{rank} A[\alpha \backslash \beta]+\operatorname{rank} A[\beta]
\end{aligned}
$$

Because of the structure of $B$

$$
\operatorname{rank} A[\alpha \backslash \beta]+\operatorname{rank} A[\beta] \leqslant \operatorname{rank} A[\alpha]
$$

It follows from (4.7) and (4.8) that

$$
\operatorname{rank} A[\alpha \mid\langle n\rangle] \leqslant \operatorname{rank} A[\alpha]
$$

but since clearly

$$
\operatorname{rank} A[\alpha \mid\langle n\rangle] \geqslant \operatorname{rank} A[\alpha]
$$

we have

$$
\operatorname{rank} A[\alpha \mid\langle n\rangle]=\operatorname{rank} A[\alpha]
$$

In a similar way we prove that

$$
\operatorname{rank} A[\langle n\rangle \mid \alpha]=\operatorname{rank} A[\alpha]
$$

Similarly we have

Proposition 4.9. Let $A$ be an $n \times n$ matrix. Then $A$ has the weak principal submatrix rank property if and only if (4.5) holds for all sets $\alpha$ such that $A[\alpha]$ is irreducible and singular.

Proof. For a nonempty $\alpha \subseteq\langle n\rangle$, denote by $m(\alpha)$ the set

$$
\alpha \cup\{j \in\langle n\rangle \backslash \alpha: A[\alpha \mid j]=0 \text { or } A[j \mid \alpha]=0\}
$$

Observe that in view of Definition 4.3, the matrix $A$ has the weak principal submatrix rank property if and only if it satisfies

$$
\operatorname{rank} A[\alpha \mid m(\alpha)]=\operatorname{rank} A[m(\alpha) \mid \alpha]=\operatorname{rank} A[\alpha]
$$


for all nonempty sets $\alpha \subseteq\langle n\rangle$. Our proof now follows exactly as the proof of Proposition 4.6, where $\langle n\rangle$ is replaced everywhere by $m(\alpha)$, using the facts that for the choice of $\beta$ in the proof of Proposition 4.6 we have

$$
\beta \subseteq \alpha \subseteq m(\beta)
$$

and

$$
\alpha \backslash \beta \subseteq \alpha \subseteq m(\alpha \backslash \beta)
$$

which imply that

$$
\beta \subseteq m(\alpha) \subseteq m(\beta)
$$

and

$$
\alpha \backslash \beta \subseteq m(\alpha) \subseteq m(\alpha \backslash \beta)
$$

Lemma 4.10. Let $A$ be an $n \times n H_{+}$-matrix in Frobenius normal form (4.1). Then $A$ has the principal submatrix rank property if and only if (4.4) holds for all sets $\alpha$ such that $A[\alpha]$ is a singular $A_{i i}, i=1, \ldots, k$.

Proof. By Lemma 3.20, it is clear that the singular irreducible principal submatrices of $\mathrm{A}$ are the singular $A_{i i}, i=1, \ldots, k$. The lemma follows from Proposition 4.6.

LEMMA 4.11. Let $A \in \mathbb{R}^{n n}$ have the principal submatrix rank property. If $A$ has a singular principal submatrix $A[\alpha]$ such that $A[\alpha]+A[\alpha]^{T}$ is positive semidefinite, then $A+A^{T}$ is singular.

Proof. Since $\mathrm{A}[\alpha]$ is singular, there exists a nonzero vector $x$ such that

$$
A[\alpha] x=0 .
$$

By Lemma 6.3 of [9]

$$
x^{T} A[\alpha]=0
$$

Because of the principal submatrix rank property it follows from (4.12) and (4.13) that

$$
A[\langle n\rangle \mid \alpha] x=0
$$


and

$$
x^{T} A[\alpha \mid\langle n\rangle]=0
$$

Hence

$$
\begin{aligned}
x^{T}\left(A+A^{T}\right)[\alpha \mid\langle n\rangle] & =x^{T} A[\alpha \mid\langle n\rangle]+x^{T} A[\langle n\rangle \mid \alpha]^{T} \\
& =x^{T} A[\alpha \mid\langle n\rangle]+(A[\langle n\rangle \mid \alpha] x)^{r}=0,
\end{aligned}
$$

which implies the singularity of $A+A^{T}$.

We now complete the characterization of Lyapunov diagonally semistable $H_{+}$-matrices.

ThEOREM 4.14. Let $A$ be an $H_{+}{ }_{-}$matrix in Frobenius normal form (4.1). Then the following are equivalent:

(i) A is Lyapunov diagonally semistable.

(ii) A has the principal submatrix rank property.

(iii) A has the weak principal submatrix rank property.

(iv) For every singular $A_{i i}$ the columns of $A_{i j}, 1 \leqslant i<j \leqslant k$, are in the span of the columns of $A_{i i}$, and the rows of $A_{l i}, 1 \leqslant l<i \leqslant k$, are in the span of the rows of $A_{i i}$.

(v) There exists a positive diagonal matrix $D$ such that the corresponding principal minors of $A$ and $A D+D A^{T}$ are either both positive or both zero.

Proof. In the case that $A$ is nonsingular, conditions (ii), (iii), and (iv) hold, since all principal minors of $A$ are positive, and conditions (i) and (v) follow from Theorem 4.2. So we assume that $A$ is singular.

(i) $\Rightarrow$ (ii): Assume that $A$ is Lyapunov diagonally semistable in Frobenius normal form (4.1). By Lemma 4.10 is enough to show that (4.4) holds where $A[\alpha]$ is any singular $A_{i i}, i=1, \ldots, k$. So let $A[\alpha]=A_{i i}$ be singular. If $i<k$ then let $j, I \leqslant j \leqslant n$, satisfy

$$
j>t \quad \text { for all } \quad t \in \alpha .
$$

Denote by $\beta$ the set $\alpha \cup\{j\}$. Observe that

$$
A[\beta]=\left[\begin{array}{cc}
A_{i i} & x \\
0 & a_{j j}
\end{array}\right],
$$

where $x=A[\alpha \mid j]$. 
principal submatrix rank property.

(ii) $\Rightarrow$ (iii): Obvious.

(iii) $\Rightarrow$ (iv) by Definition 4.3 .

(ii) $\Leftrightarrow$ (iv) by Lemma 4.10 .

(ii) $\Rightarrow(v)$ : Let $A$ have the principal submatrix rank property. We prove (v) by induction on the number $k$ of irreducible diagonal blocks in the form (4.1). For $k=1$, if $A$ is nonsingular then $A$ is Lyapunov diagonally stable by Theorem (4.2). If $A$ is singular, then by Theorem 3.19 there exists a positive diagonal matrix $D$ such that $A D+D A^{T}$ is positive semidefinite and $A$ is sign similar to $M(A)$. Hence $A D+D A^{T}$ is an irreducible $H_{+}$-matrix, and by Lemma 3.20 all the principal minors of $A$ and of $A D+D A^{T}$ of order less than $n$ are positive. The singularity of $A D+D A^{T}$ follows from Lemma 6.3 of [9] (also from Theorem 3.19).

Assume that the claim holds for $k<m$, and let $k=m$. We write $A$ as

$$
A=\left[\begin{array}{cc}
B_{11} & B_{12} \\
0 & B_{22}
\end{array}\right]
$$

where

$$
B_{11}=\left[\begin{array}{cccccc}
A_{11} & \cdot & \cdot & \cdot & \cdot & A_{1, m-1} \\
0 & A_{22} & & & & \cdot \\
\cdot & \cdot & \cdot & \cdot & & \cdot \\
\cdot & & \cdot & \cdot & \cdot & \cdot \\
0 & \cdot & \cdot & \cdot & 0 & A_{m-1, m-1}
\end{array}\right] \text { and } B_{22}=A_{m m}
$$

Let $\beta \subseteq\langle n\rangle$ be the set such that $B_{11}=A(\beta)$, and let $\gamma=\langle n\rangle \backslash \beta$. By the inductive assumption there exist positive diagonal matrices $D_{1}$ and $D_{2}$ such that

$$
\begin{cases}\forall \delta \subseteq \gamma & \text { signdet } A[\delta]=\operatorname{sign} \operatorname{det}\left(A D_{1}+D_{1} A^{T}\right)[\delta] \\ \forall \delta \subseteq \beta & \text { signdet } A[\delta]=\operatorname{sign} \operatorname{det}\left(A D_{2}+D_{2} A^{T}\right)[\delta]\end{cases}
$$

Define $D=D_{1} \oplus \varepsilon D_{2}$, where $\varepsilon$ is a positive scalar, and let $C=A D+D A^{T}$. Consider a principal submatix $C[\alpha]$. If either $\alpha \cap \gamma=\varnothing$ or $\alpha \cap \beta=\varnothing$, then our claim follows from (4.20). Otherwise, if $A[\alpha]$ is singular, then either $A[\alpha \cap \beta]$ or $A[\alpha \cap \gamma]$ is singular. In this case we apply Lemma 4.11 to $(A D)[\alpha]$, and get that $C[\alpha]$ is singular. If $\operatorname{det} A[\alpha]$ is positive, then so are 
Since $A$ is Lyapunov diagonally semistable if and only if $A D$ is Lyapunov diagonally semistable for each positive diagonal matrix $D$, and since the principal submatrix rank property is invariant under multiplication by a nonsingular diagonal matrix, we may assume that $A+A^{T}$ is positive semidefinite. Therefore $A[\beta]+A[\beta]^{T}$ is positive semidefinite, which is equivalent to

$$
u^{T} A[\beta] u \geqslant 0 \quad \text { for every vector } \quad u \text {. }
$$

Let $v$ be a nonzero vector satisfying

$$
v^{T} A_{i i}=0
$$

and let $u$ be the nonzero vector $\left[\begin{array}{l}v \\ \varepsilon\end{array}\right]$, where $\varepsilon$ is any real number. Observe that

$$
u^{T} A[\beta] u=\varepsilon\left(v^{T} x+\varepsilon a_{j j}\right)
$$

If $v^{T} x \neq 0$, then for $\varepsilon$ small enough with sign opposite to that of $v^{T} x$, the expression (4.18) will be negative, which is a contradiction to (4.16). Thus

$$
v^{T} x=0
$$

Denote by $l$ the order of $A_{i i}$. By Lemma 3.20,

$$
\operatorname{rank} A_{i i}=l-1
$$

and it follows from (4.17) that the columns of $A_{i i}$ span the subspace of $\mathbb{C}^{l}$ which is orthogonal to $v$. Therefore, by (4.19), $x=A[\alpha \mid j]$ is a linear combination of the columns of $A[\alpha]$. Since this holds for any $j$ satisfying (4.15), we have proved that

$$
\operatorname{rank} A[\alpha \mid\langle n\rangle]=\operatorname{rank} A[\alpha]
$$

Considering indices $j, 1 \leqslant j \leqslant n$, satisfying $j<t$ for all $t \in \alpha$, we show in a similar way that

$$
\operatorname{rank} A[\langle n\rangle \mid \alpha]=\operatorname{rank} A[\alpha]
$$

So (4.4) is satisfied for every $A[\alpha]$ that is a singular $A_{i i}$, and thus $A$ has the 
$\operatorname{det} A[\alpha \cap \beta]$ and $\operatorname{det} A[\alpha \cap \gamma]$. In this case $\operatorname{det} C[\alpha]$ is a polynomial in $\varepsilon$, where the lowest power $\varepsilon^{|\alpha \cap \beta|}$ has the positive coefficient $\operatorname{det} A[\alpha \cap$ $\beta] \operatorname{det} A[\alpha \cap \gamma]$. Therefore, for $\varepsilon$ sufficiently small, $\operatorname{det} C[\alpha]$ will be positive. Since the number of the subsets of $\langle n\rangle$ is finite, we can choose $\varepsilon$ small enough such that $D$ is the required positive diagonal matrix.

(v) $\Rightarrow$ (i): Obvious, since $A$ is a $P_{0}$-matrix.

REMARK. Using arguments similar to those in the first part of the proof of Theorem 4.14, one can prove the known results that a positive semidefinite matrix has the principal submatrix rank property.

The following proposition is closely related to known results as in Lemma 5 of [13]. We give a proof for the sake of completeness.

Proposition 4.21. Let $A \in \mathbb{R}^{n n}$ be an M-matrix in Frobenius normal form (4.1). Then $A$ has the principal submatrix rank property if and only if $A_{i i}$ singular implies that $A_{i j}=0, A_{j i}=0, j \neq i, j=1, \ldots, k$.

Proof. The "if" part is obvious. Conversely, assume that $A$ has the principal submatrix rank property, and let $A[\alpha]=A_{i i}$ be singular. Let $x$ be a positive vector satisfying

$$
A[\alpha] x=0
$$

By (4.4)

$$
A[\langle n\rangle \backslash \alpha \mid \alpha] x=0
$$

But since $x$ is positive and $A[\langle n\rangle \backslash \alpha \mid \alpha]$ is nonpositive, then necessarily $A[\langle n\rangle \backslash \alpha \mid \alpha]=0$. The proof that $A[\alpha \mid\langle n\rangle \backslash \alpha]=0$ is essentially the same.

In view of Proposition 4.21, Theorem 4.14 generalizes Theorem 0.1.

We now show that the implication $(v) \Rightarrow$ (ii) in Theorem 4.14 holds in general.

Lemma 4.22. Let $A \in \mathbb{R}^{n n}$, and suppose that $A+A^{T}$ is positive semidefinite. Then rank $A$ equals the maximum of the orders of nonsingular principal submatrices of $A$.

Proof. By Corollary III.1 of [6], the elementary divisors belonging to the eigenvalue 0 of $A$ are linear. Hence, the nullity of $A$ equals the algebraic 
multiplicity of 0 as an eigenvalue of $A$. Let the characteristic polynomial of $A$ be $\sum_{r=0}^{\prime \prime} c_{n-r} \lambda^{r}$. Thus, rank $A$ equals the maximum $k$ such that $c_{k} \neq 0$. Since $(-1)^{k} c_{k}$ is the sum of all principal minors of $A$ of order $k$, and since $A$ is a $P_{0}$-matrix (being Lyapunov diagonally semistable), the results follows.

Theorem 4.23. Let $A \in \mathbb{R}^{n n}$, and assume that there exists a positive diagonal matrix $D$ such that the corresponding principal minors of $A$ and $A D+D A^{T}$ are either both positive or both zero. Then $A$ has the principal submatrix rank property.

Proof. Since the principal submatrix rank property is invariant under multiplication by a nonsingular diagonal matrix, we may assume, without loss of generality, that the matrix $D$ may be chosen to be the identity matrix (otherwise we apply our assertion for $A D$ ). By Lemma 4.22 the rank of $A$ equals the maximum of the orders of nonsingular principal submatrices of $A$. Since the same holds for any principal submatrix of $A$, it follows from the assumptions of the theorem that

$$
\operatorname{rank} A[\beta]=\operatorname{rank}\left(A+A^{T}\right)[\beta] \quad \text { for all } \beta \subseteq\langle n\rangle
$$

We now choose $\alpha \subseteq\langle n\rangle$, and we shall show that (4.4) holds. If $A[\alpha]$ is nonsingular then there is nothing to prove. If $A[\alpha]$ is singular then by Lemma 3.22

$$
N(A[\alpha])=N\left(A[\alpha]^{T}\right) \subseteq N\left(\left(A+A^{T}\right)[\alpha]\right)
$$

and it follows from (4.24) that

$$
N(A[\alpha])=N\left(A[\alpha]^{T}\right)=N\left(\left(A+A^{T}\right)[\alpha]\right)
$$

Since, as is well known, for any square matrix $X$,

$$
N(X)^{\perp}=\operatorname{span}\left(X^{T}\right)
$$

where $V^{\perp}$ is the orthogonal complement of a vector space $V$, we have

$$
\operatorname{span}(A[\alpha])=\operatorname{span}\left(A[\alpha]^{T}\right)=\operatorname{span}\left(\left(A+A^{T}\right)[\alpha]\right) .
$$

If for all $j, j=1, \ldots, n, j \notin \alpha, A[\alpha \mid j]$ and $A[j \mid \alpha]^{T}$ are in $\operatorname{span}(A[\alpha])$, then 
(4.4) holds. Otherwise, assume that for some $j, j=1, \ldots, n, j \notin \alpha$,

(4.27) $A[\alpha \mid j] \notin \operatorname{span}(A[\alpha])$ or $A[j \mid \alpha]^{T} \notin \operatorname{span}(A[\alpha])$.

Observe that, by (4.26), $\left(A+A^{T}\right)[\alpha]$ is a singular principal submatrix of the positive semidefinite matrix $A+A^{T}$. As such, it is well known that

(4.28) $A[\alpha \mid j]+A[j \mid \alpha]^{T}=\left(A+A^{T}\right)[\alpha \mid j] \in \operatorname{span}\left(\left(A+A^{T}\right)[\alpha]\right)$.

By (4.26), (4.27), and (4.28) we have

$A[\alpha \mid j] \notin \operatorname{span}(A[\alpha])$,

and

$$
A[j \mid \alpha]^{T} \notin \operatorname{span}\left(A[\alpha]^{T}\right) .
$$

Let $\gamma=\alpha \cup\{j\}$. It follows from (4.29) that

$$
\operatorname{rank} A[\alpha \mid \gamma]=\operatorname{rank} A[\alpha]+1
$$

and from (4.30) that

$$
\operatorname{rank} A[\gamma]=\operatorname{rank} A[\alpha \mid \cdot \gamma]+1
$$

So, by (4.31) and (4.32)

$$
\operatorname{rank} A[\gamma]=\operatorname{rank} A[\alpha]+2
$$

But, by (4.28),

$$
\operatorname{rank}\left(A+A^{T}\right)[\alpha \mid \gamma]=\operatorname{rank}\left(A+A^{T}\right)[\alpha]
$$

and therefore

$$
\operatorname{rank}\left(A+A^{T}\right)[\gamma] \leqslant \operatorname{rank}\left(A+A^{T}\right)[\alpha]+1
$$

By (4.24)

$$
\operatorname{rank} A[\alpha]=\operatorname{rank}\left(A+A^{T}\right)[\alpha]
$$


So it follows from (4.33) and (4.34) that

$$
\operatorname{rank}\left(A+A^{T}\right)[\gamma]<\operatorname{rank} A[\gamma]
$$

which is a contradiction to (4.24). Hence, (4.27) is false and (4.4) holds.

Another implication that holds in general is (i) $\Rightarrow$ (iii).

THEOREM 4.35. Let $A \in \mathbb{R}^{n n}$. If $A$ is a Lyapunov diagonally semistable, then $A$ has the weak principal submatrix rank property.

Proof. Let $\alpha$ be a nonempty subset of $\langle n\rangle$, and let $j \in\langle n\rangle \backslash \alpha$ be such that $A[j \mid \alpha]=0$. We denote by $\beta$ the set $\alpha \cup\{j\}$.

Let $D$ be a Lyapunov scaling factor of $A$. Clearly, $C=A[\beta] D[\beta]+$ $D[\beta] A[\beta]^{r}$ is positive semidefinite. Since $A[\alpha]$ is singular, by Lemma 3.22 the matrix

$$
B=A[\alpha] D[\alpha]+D[\alpha] A[\alpha]^{T}
$$

is a singular principal submatrix of the positive semidefinite matrix $C$. Therefore,

$$
d_{j j} A[\alpha \mid j]=C[\alpha \mid j] \in \operatorname{span} B
$$

It is known that if $V_{1}$ and $V_{2}$ are subspaces of a given vector space $V$, then

$$
V_{1} \subseteq V_{2} \quad \Rightarrow \quad V_{2}^{\perp} \subseteq V_{1}^{\perp}
$$

By Lemma 3.22, $N\left(A^{T}\right) \subseteq N\left(A D+D A^{T}\right)$, and hence by (4.25), (4.36), and (4.37) we have, since $B$ is symmetric,

$$
d_{j j} A[\alpha \mid j] \in \operatorname{span}(B)=\operatorname{span}\left(B^{T}\right) \subseteq \operatorname{span}(A[\alpha]) .
$$

Similarly we show that $A[\alpha \mid j]=0$ implies that $A[j \mid \alpha]^{T} \in \operatorname{span}\left(A[\alpha]^{T}\right)$.

In view of Theorems 4.23 and 4.35 and the obvious implications (ii) $\Rightarrow$ (iii) $\Rightarrow$ (iv) and (v) $\Rightarrow$ (i), we have in general

$$
\begin{aligned}
(\mathrm{i}) & \Rightarrow(\mathrm{iii}),(\mathrm{iv}), \\
(\mathrm{ii}) & \Rightarrow(\mathrm{iii}),(\mathrm{iv}), \\
(\mathrm{iii}) & \Rightarrow(\mathrm{iv}), \\
(\mathrm{v}) & \Rightarrow(\mathrm{i}),(\mathrm{ii}),(\mathrm{iii}),(\mathrm{iv}) .
\end{aligned}
$$


These implications can be summarized by the following diagram:

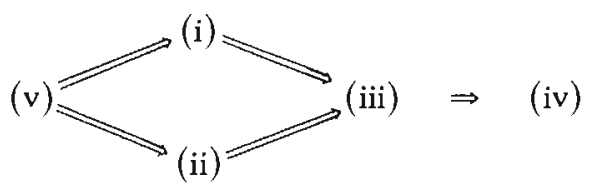

We give three examples demonstrating that none of the other possible implications hold in general, not even for $P_{0}$-matrices. Our examples involve nonsingular matrices. In order to have examples of singular matrices it is enough to add a zero row and a zero column to each of the matrices considered below.

EXAMPLE 4.39. The matrix

$$
A=\left[\begin{array}{lll}
0 & 1 & 0 \\
0 & 0 & 1 \\
1 & 0 & 0
\end{array}\right]
$$

demonstrates that (iv) $\Rightarrow$ (iii). Hence in view of (4.38) we have (iv) $\nRightarrow(i),(i i),(v)$.

Example 4.40. Consider the $P$-matrix

$$
A=\left[\begin{array}{llll}
1 & 2 & 0 & 0 \\
0 & 1 & 2 & 0 \\
2 & 0 & 1 & 2 \\
0 & 0 & 0 & 1
\end{array}\right]
$$

with $A_{11}=A[1,2,3]$. The matrix $A_{11}$ is Lyapunov diagonally semistable, since

$$
A_{11}+A_{11}^{T}=\left[\begin{array}{lll}
2 & 2 & 2 \\
2 & 2 & 2 \\
2 & 2 & 2
\end{array}\right]
$$

Observe that $A$ has the principal submatrix rank property, since all its principal minors are positive. Assume that there exists a positive diagonal 
matrix $D=\operatorname{diag}\left\{d_{1}, d_{2}, d_{3}, d_{4}\right\}$ such that

$$
C=A D+D A^{T}=2\left[\begin{array}{cccc}
d_{1} & d_{2} & d_{1} & 0 \\
d_{2} & d_{2} & d_{3} & 0 \\
d_{1} & d_{3} & d_{3} & d_{4} \\
0 & 0 & d_{4} & d_{4}
\end{array}\right]
$$

is positive semidefinite. It is easy to verify that

$$
\begin{aligned}
& \operatorname{det} C[1,2] \geqslant 0 \quad \Rightarrow \quad d_{1} \geqslant d_{2}, \\
& \operatorname{det} C[1,3] \geqslant 0 \quad \Rightarrow \quad d_{3} \geqslant d_{1}, \\
& \operatorname{det} C[2,3] \geqslant 0 \quad \Rightarrow \quad d_{2} \geqslant d_{3} .
\end{aligned}
$$

The last three inequalities imply

$$
d_{1}=d_{2}=d_{3}
$$

But then

$$
\operatorname{det} C[2,3,4]=-8 d_{2} d_{4}^{2}<0,
$$

which is a contradiction to $C$ 's being positive semidefinite. Thus $A$ is not Lyapunov diagonally semistable. Hence, this example demonstrates that even for $P$-matrices, (ii), (iii) $\nRightarrow($ i). By (4.38) (ii), (iii) $\nRightarrow(v)$.

EXAMPLE 4.41. Let

$$
A=\left[\begin{array}{rr}
0 & 1 \\
-1 & 0
\end{array}\right]
$$

Clearly, $A$ does not have the principal submatrix rank property, but $A$ is Lyapunov diagonally semistable, since $A+A^{T}=0$. Also, it follows from Theorem 6.17 of [9] that the identity matrix is the unique Lyapunov scaling factor of $A$, and since the determinant of $A$ is positive, $A$ does not fulfill (v). Hence, even for $P_{0}$-matrices, (i) $\Rightarrow$ (ii). This example also shows that (iii) $\neq$ (ii). Hence, by (4.38) we have (i), (iii) $\neq(\mathrm{v})$. 
5. APPLICATIONS TO NUMERICAL ABSCISSAS OF REAL MATRICES

Definition 5.1. Let $A \in \mathbb{R}^{n n}$.

(i) The spectral abscissa $\sigma(A)$ of $A \in \mathbb{R}^{n n}$ is defined to be

$$
\sigma(A)=\max \{\operatorname{Re} \lambda: \lambda \in \operatorname{spec}(A)\} .
$$

(ii) The symmetric part of $A$, denoted by $\operatorname{Re}(A)$, is defined to be

$$
\operatorname{Re}(A)=\frac{A+A^{T}}{2}
$$

(iii) The numerical abscissa of $A$ is defined to be

$$
\nu(A)=\sigma(\operatorname{Re}(A)) .
$$

Definition 5.2. Let $A, B \in \mathbb{R}^{n n}$. Then $A$ and $B$ are diagonally similar $(A \sim B)$ if there exists a nonsingular diagonal matrix $D \in \mathbb{R}^{n n}$ such that $B=D^{-1} A D$. The set of all matrices in $\mathbb{R}^{n n}$ diagonally similar to $A$ will be denoted by $\mathscr{D}(A)$.

Note that the symbol was used more restrictively in [8]. In this section we apply the results of the previous sections to compare the numerical abscissas of matrices $B$ diagonally similar to a given $A \in \mathbb{R}^{n n}$ to the spectral abscissas of $A$ and $|A|$. It is easy to show that $\nu(B)$ is in fact also the maximum of real parts of elements of the field of values $V(B)$, where as usual

$$
V(B)=\left\{x^{*} B x: x \in \mathbb{C}^{n}, x^{*} x=1\right\} .
$$

This remark yields the well-known inequality $\sigma(A) \leqslant \nu(A)$ for $A \in \mathbb{R}^{n n}$, and hence, since $\operatorname{spec}\left(D^{-1} A D\right)=\operatorname{spec}(A)$ for a nonsingular diagonal matrix $D$, it follows that

$$
\sigma(A) \leqslant \nu(B) \text { if } B \in \mathscr{D}(A) .
$$

Further, since $|A| \geqslant 0$, it follows immediately from Perron-Frobenius that

$$
\sigma(|A|) \text { is the spectral radius of }|A| \text {. }
$$


It is easy to reformulate our results on Lyapunov diagonal stability in terms of diagonal similarity. We shall not do this in detail, but we furnish the required means in our next lemma. Let $D$ be a positive diagonal matrix. Clearly, the matrix $A D^{2}+D^{2} A^{T}$ is positive (semi)definite if and only if $D^{-1} A D$ has positive (semi)definite symmetric part. Thus the next lemma is an immediate consequence of the following definition.

Definition 5.5. Let $A \in \mathbb{R}^{n n}$. We set

$$
H(A)=\sigma(|A|) I-A
$$

Lemma 5.6. Let $A \in \mathbb{R}^{n n}$, and let $D$ be a nonsingular diagonal matrix. Then

(i) $H(A)$ is an $H_{+}-$matrix.

(ii) $H(A)$ is a nonsingular $H_{+}-$matrix if and only if $\sigma(A)<\sigma(|A|)$.

(iii)

$$
\sigma(A) \leqslant \nu\left(D^{-1} A D\right) \leqslant \sigma(|A|)
$$

if and only if $D^{2}$ is a Lyapunov scaling factor of $H(A)$.

(iv): Let $\varepsilon>0$. Then

$$
\sigma(A) \leqslant \nu\left(D^{-1} A D\right) \leqslant \sigma(|A|)+\varepsilon
$$

if and only if $D^{2}$ is a Lyapunov scaling factor for $H(A)+\varepsilon I$.

(v)

$$
\sigma(A) \leqslant \nu\left(D^{-1} A D\right)<\sigma(|A|)
$$

if and only if $H(A) D^{2}+D^{2} H(A)^{T}$ is positive definite.

Proof. (i): Since $M(H(A))=\sigma(|A|) I-|A|=M(|A|)$, and the latter matrix is an $M$-matrix, it follows that $H(A)$ is an $H$-matrix. Furthermore, $H(A)$ is an $H_{+}$-matrix, since $\sigma(|A|) \geqslant\left|a_{i i}\right|, i=1, \ldots, n$.

(ii): follows from $\sigma(A) \leqslant \sigma(|A|)$.

(iii): Let $D$ be a nonsingular diagonal matrix. By (5.3),

$$
\nu\left(D^{-1} A D\right) \leqslant \sigma(|A|)
$$


is equivalent to (5.7). By Sylvester's inertia Theorem, $D^{2}$ is a Lyapunov scaling factor for $H(A)$ if and only if $D^{-1} H(A) D+\left(D^{-1} H(A) D\right)^{T}=$ $D^{-1}\left(H(A) D^{2}+D^{2} H(A)\right) D^{-1}$ is positive semidefinite. But this holds if and only if $(5.10)$ is satisfied.

(iv): Similar to (ii).

(v): Similar to (iii).

Theorem 5.11. Let $A \in \mathbb{R}^{n n}$. Then

$$
\sigma(A) \leqslant \inf \{\nu(B): B \sim A\} \leqslant \sigma(|A|) .
$$

Proof. In view of Lemma 5.6(i), $H(A)+\varepsilon I$ is nonsingular $H_{+}$-matrix for all positive $\varepsilon$. Hence by Theorem 4.2 , there is a Lyapunov scaling factor for $H(A)+\varepsilon I$. By Lemma 5.6(iv) there exists a nonsingular $D$ for which $\sigma(A) \leqslant$ $\nu\left(D^{-1} A D\right) \leqslant \sigma(|A|)+\varepsilon$, and (5.12) now follows.

We note that the inequality (5.12) does not imply the existence of a matrix $B \in \mathscr{D}(A)$ for which

$$
\sigma(A) \leqslant \nu(B) \leqslant \sigma(|A|)
$$

in the case that $\sigma(A)=\sigma(|A|)$. For let

$$
A=\left[\begin{array}{ll}
0 & 1 \\
0 & 0
\end{array}\right]
$$

Then $\sigma(A)=\sigma(|A|)=0$, but for all nonsingular diagonal $D, \nu\left(D^{-1} A D\right)>0$. Our next proposition clarifies this situation.

Proposition 5.14. Let $A \in \mathbb{R}^{n n}$. There exists a $B \in \mathscr{D}(A)$ such that (5.13) holds if and only if $\sigma(|A|) I-A$ has the (weak) principal submatrix rank property.

Proof. By Lemma 5.6(iii), (5.13) is equivalent to the existence of a Lyapunov scaling factor for $H(A)$. The proposition now follows from Theorem 4.14.

We do not know in general under what conditions the infimum in (5.12) is achieved. But we have some partial results of interest. 
THEOREM 5.15. Let $A \in \mathbb{R}^{n n}$. Then the following are equivalent:

(i) The second equality holds in (5.12).

(ii) Both equalities hold in (5.12).

Proof. (i) $\Rightarrow$ (ii): Assume that

$$
\sigma(A)<\sigma(|A|)
$$

Then the $H_{+}$-matrix $H(A)$ is nonsingular by Lemma 5.6(ii). Hence, by Theorem 4.2, $H(A)$ is Lyapunov diagonally stable. Thus, by Lemma 5.6(v) there is a $B \in \mathscr{D}(A)$ with $\nu(B)<\sigma(|A|)$. Hence the second equality in (5.12) implies $\sigma(A)=\sigma(|A|)$.

(ii) $\Rightarrow$ (i): Trivial.

Observe that it is necessary and sufficient for either of the equivalent conditions in Theorem 5.15 to be satisfied that there should exist a block $A_{i i}$ in the Frobenius normal form of $A$ with $\sigma\left(A_{i i}\right)=\sigma(|A|)$.

For irreducible $A$ we have some additional conditions.

THEOREM 5.16. Let $A \in \mathbb{R}^{n n}$ be irreducible. Then the following are equivalent:

(i) The matrix $\sigma(|A|) I-A$ is singular.

(ii) The second equality holds in (5.12).

(iii) Both equalities hold in (5.12).

(iv) $A$ is sign similar to a nonnegative matrix.

(v) There is a nonnegative matrix in $\mathscr{D}(A)$.

Proof. (i) $\Leftrightarrow$ (ii) by Lemma 5.6(ii).

(ii) $\Leftrightarrow$ (iii) by Theorem 5.15 .

(iii) $\Leftrightarrow$ (iv) by Theorem 2.6.

(iv) $\Leftrightarrow(v)$ is trivial.

We remark that the first equality in (5.12) does not imply the second. An example is furnished by $A=[-1] \in \mathbb{R}^{11}$ and by

$$
B=\left[\begin{array}{rr}
0 & 1 \\
-1 & 0
\end{array}\right]
$$

For this example it is easily seen that $\sigma(B)=\nu(B)=0<1=\sigma(|B|)$. 
Theorem 5.17. Let $A \in \mathbb{R}^{n n}$. Then there exists a unique positive diagonal matrix $D$ satisfying (5.7) if and only if $A$ is irreducible and $A$ is sign similar to a nonnegative matrix.

Proof. By Lemma 5.6(iii) there exists a unique positive diagonal matrix $D$ satisfying (5.7) if and only if $H(A)$ has a unique Lyapunov scaling factor. By Remark 3.24 and by Theorem 3.25 this is equivalent to the irreducibility and singularity of $H(A)$. Our theorem now follows from Theorem 5.15.

\section{REFERENCES}

1 M. Araki and B. Kondo, Stability and transient behaviour of composite nonlinear systems, IEEE Trans. Automat. Control AC-17:537-541 (1972).

2 G. P. Barker, A. Berman, and R. J. Plemmons, Positive diagonal solutions to the Lyapunov equations, Linear and Multilinear Algebra 5:249-256 (1978).

3 A. Berman and D. Hershkowitz, Matrix diagonal stability and its implications, SIAM J. Algebraic Discrete Methods 4:377-382 (1983).

4 A. Berman and R. J. Plemmons, Nonnegative Matrices in the Mathematical Sciences, Academic, New York, 1979.

5 A. Berman, R. S. Varga, and R. C. Ward, ALPS: Matrices with nonpositive off-diagonal entries, Linear Algebra Appl. 21:233-244 (1978).

6 D. Carlson and H. Schneider, Inertia theorems for matrices: The semidefinite case, J. Math. Anal. Appl. 6:430-446 (1963).

7 G. W. Cross, Three types of matrix stability, Linear Algebra Appl. 20:253-263 (1978).

8 S. Friedland, D. Hershkowitz, and H. Schneider, Matrices whose powers are $M$-matrices or Z-matrices, to appear.

9 D. Hershkowitz and H. Schneider, Scalings of vector spaces and the uniqueness of Lyapunov scaling factors, Linear and Multilinear Algebra, to appear.

10 R. Horn and C. R. Johnson, Matrix Analysis, Cambridge U.P., to appear.

11 M. Neumann and R. J. Plemmons, Backward error analysis for linear systems associated with inverses of $H$-matrices, BIT 24:102-112 (1984).

12 A. Ostrowski, Über die Determinanten mit überwiegender Hauptdiagonale, Comment. Helv. Math. 10:69-96 (1937).

$13 \mathrm{H}$. Schneider, The elementary divisors, associated with 0 , of a singular $M$-matrix, Proc. Edinburgh Math. Soc. (2) 10:108-122 (1956).

14 L. Tartar, Une nouvelle characterization des $M$-matrices, Rev. Française Informat. Recherche Operationnelle 5:127-128 (1971).

15 H. Wielandt, Unzerlegbare, nicht-negative Matrizen, Math. Z. 52:642-648 (1950). 